\title{
Smart Integrated Adaptive Centralized Controller for Islanded Microgrids under Minimized Load Shedding
}

\author{
M. Karimi ${ }^{1}$, R. Azizipanah-Abarghooee ${ }^{1}$, H. Uppal ${ }^{1}$, Q. Hong $^{2}$, C. Booth ${ }^{2}$, and V. Terzija ${ }^{1}$ \\ ${ }^{1}$ The University of Manchester, UK $\quad{ }^{2}$ University of Strathclyde, UK
}

\begin{abstract}
In this paper, a smart integrated adaptive centralized controller is proposed for monitoring and controlling integrated renewable energy sources (RESs), both for intentional and unintentional islanding modes of operation for microgrids, as well as, for a variable range of transient load shedding and fault scenarios corresponding to electrical power system outages. It is demonstrated that the proposed smart adaptive controller is capable of instructing fast frequency response by proper coordination of the dispatch of RESs units such as, mini-hydro, Photovoltaic (PV), Battery Energy Storage System (BESS) and standby diesel generators. In particular, the BESS used as power reserve, at the early stage of fault events can prevent detrimental and uncontrollable system frequency decline and the extent of load shedding. In summary, the performance of a centralized controller in terms of a fast frequency response recovery feature is validated for an actual microgrid distribution network of Malaysia. The demonstration of this intelligent control scheme highlights the advantage of utilizing the fast power recovery response of energy storage and standby generator, which fulfil the criteria for minimal load shedding from the main grid, during the unintentional microgrid islanding conditions.
\end{abstract}

Index Terms - Frequency response, microgrid, renewable energy sources, battery energy storage, islanding operation, transient load shedding, distribution network, adaptive centralized controller.

\section{INTRODUCTION}

With the wider integration of Renewable Energy Sources (RES) to the power networks, especially in distribution levels, operational reliability and resilience of microgrids have attracted increasing interest for enhanced integration in future smart grid architectures of power systems [1]. The microgrids in distribution networks can be operated autonomously for a grid-connected or islanded mode to improve reliability and quality of power delivery [2]. In these microgrids, the RESs are either small-scale synchronous generators or converter-interfaced sources (e.g. PV, energy storage, wind, etc.) resulting in a small inertia of the network [3]. In addition, whereas the renewable energy resources are mostly intermittent in nature, it is assumable that the microgrid will require importing power from the main grid when the renewable resource and/or the synchronous generation are not sufficient for supplying and sustaining local loads. Under such circumstances, if an unintentional islanding event occurs, the local network will be at risk of experiencing significant frequency degradation in a short period of time with the added complexity of small inertia of the power system [4].

To avoid the collapse of the system, load shedding schemes are widely used to curtail loads with low priorities to rebalance the supply and demand, where a variety of conventionally load shedding schemes to cope with the above scenarios have been reported in [5-9]. A dynamic load shedding considered and formulated as a stochastic optimisation problem to economically optimise the islanded microgrid using Markov decision model is one of its kind [5]. A new strategy based on optimal non-linear predictive control model is recently proposed for an islanded $\mathrm{MG}$ to appropriately conserve the grid stability by managing the centralised load shedding mechanism with the integration of battery energy storage [6]. A centralized coordination framework on the basis of three supervisory control stages is also suggested to manage the state of charge equalization among the energy storage units for avoiding uneven degradation, curtailment of the active power of generating units for preventing storage system's overcharge, and load shedding strategies, for avoiding deep discharge of the storage systems [7]. A centralized adaptive under-frequency load shedding architecture using a distribution state estimator is also recently validated in order to enhance the frequency stability of islanded MG [8]. Apart from the centralised approaches; the decentralised controllers have been also investigated, for example, a new coordination control methodology based on two-level i.e. primary and secondary phase controls is also demonstrated to effectively manage energy storage assets, photovoltaic (PV) units and controllable dynamic load units in a single phase low voltage MGs [9]. In particular, a V-I droop mechanism is of interest in the deployment of primary control levels, while a distributed strategy is derived for the secondary one, to simultaneously regulate the voltage of a $\mathrm{MG}$ and to manage state of charge (SoC) power among the storage units.

Since the main challenge of the current and future topologies of MGs is circumventing the lack of system's inertia, designing centralised controllers especially for load shedding scheme is difficult to achieve, as to trigger sufficiently fast response while minimizing the disconnection. Furthermore, even under load disconnection based on conventional low-priority selection, it can still bring significant inconvenience to the customers due to the interruption to the supply which is highly undesirable. This paper presents a solution for operationally sustaining a microgrid with minimized load shedding through adaptively 
dispatching energy storage and standby diesel generator power assets in microgrid.

For a real-time application point of view, energy storage devices are widely available in microgrid, e.g. renewable energy distribution hubs, electrical charging power points, etc., whereas standby diesel generators are generally available in large load centres, e.g. hospitals, data centre and large commercial buildings, etc. [10]. One key advantage of energy storage is that it can be instructed to supply power and/or energy surplus to the distribution network, as a very fast transient response. Being utilized effectively, the energy storage assets can allow a fast response to restore frequency when the microgrid is suddenly disconnected from the main network. However, as energy devices can normally support power delivery to the network for a limited period of time only, due to the constraints of their overall capacity and charging/discharging rates, this can be critical to feasibly implement without an intelligent power delivery control scheme. On the other hand, standby generators generally allow longer term of operation to generate power to support the microgrid, but it requires a certain period of time for startups and synchronization. In this paper, thereby a smart integrated centralized controller with adaptive control strategy is proposed to proper coordinate the dispatch of energy storage units and diesel generators for optimal frequency response. The system is validated in a realistic distribution network model. The integration of energy storage and the standby generator as auxiliary power sources is presented as a main feature of the controller, which can be used to sustain a faster frequency response during unintentional islanding in microgrids, while minimizing the need for load shedding.

\section{The Proposed CENTRALIZED CONTROLlER}

The proposed method requires the measurement of frequency and Rate of Change of Frequency (RoCoF) in the microgrid. In this work, these quantities are measured using a Phasor Measurement Unit (PMU). If RoCoF drops suddenly, it means that there is a sudden disturbance in the system so minimum value of the RoCoF will be captured by proposed controller used as set point, which is usually occurring within the first $20 \mathrm{~ms}$ elapsed time of the event. It must be noted; algorithmic implementation proposed here is variably independent to the $20 \mathrm{~ms}$ time and is capable of capturing the first minimum value of the RoCoF, even after the event. This minimum value of $\mathrm{RoCoF}$ is then used to calculate and estimate the disturbance size of the event according to the following equation [11]:

$$
\Delta P=\frac{2 \times H}{f_{n}} \times R o C o F
$$

where, $\Delta P$ is the power imbalance, $H$ is the inertia constant of the microgrid, $f_{n}$ is the rated frequency, $R o C o F$ is the rate of change of frequency of microgrid which is measured by PMU.

According to the simplest expression of the swing equation [11], imbalanced power set point is estimated and the proposed controller requests the equivalent amount of required power from available RESs in the system, where the choice of selectivity of RESs is based on the fast response of inverter based RESs, to inject the required amount of imbalanced active power against the set point. In our proposed methodology, instead of load reduction in the system, new technology integration such as renewable energy sources with fast response helps to compensate the lack of active power generation in the system. Under these circumstances, the fast responsiveness of the inverter-based renewable energy sources acts a surplus power buffer which facilitates a fast recovery of the system frequency. The proposed algorithm of adaptive centralized controller is depicted in Fig. 2.



Fig. 2. The proposed algorithm for smart integrated adaptive centralized controller

Following the activation of power injection from energy storage asset, the proposed controller also starts the standby diesel generator till its synchronization with the islanded microgrid power set points. The diesel generator is utilized as a standby energy reserve due to limitation on power capacity and duration of the inverter-based generation which is BESS. Whenever, diesel generator becomes synchronized with islanded microgrid, based on all three parameters in Table 1 [12], the controller sends the control signal to close the diesel generator circuit breaker. Simultaneously, the injected power of inverter-based generation is controlled to be stopped when required. Thereby, the required amount of active power in an islanded operation of microgrid can switch from inverterbased generation to diesel generator, based on the set point of power required, and vice versa controls the undesirable decline in system frequency. 
Table 1. Synchronization parameter limits for synchronous interconnection [12]

\begin{tabular}{|c|c|c|c|}
\hline $\begin{array}{c}\text { Aggregate } \\
\text { rating of RES } \\
\text { unit (kVA) }\end{array}$ & $\begin{array}{c}\text { frequency } \\
\text { difference } \\
(\mathbf{\Delta f}, \mathbf{H z})\end{array}$ & $\begin{array}{c}\text { Voltage } \\
\text { difference } \\
(\mathbf{\Delta V}, \boldsymbol{\%})\end{array}$ & $\begin{array}{c}\text { Phase angle } \\
\text { difference } \\
\left(\mathbf{\Delta} \boldsymbol{\varphi},{ }^{\circ}\right)\end{array}$ \\
\hline $0-500$ & 0.3 & 10 & 20 \\
\hline$>500-1500$ & 0.2 & 5 & 15 \\
\hline$>1500-10000$ & 0.1 & 3 & 10 \\
\hline
\end{tabular}

\section{CASE STUDY}

\section{A. Disribution System Network Model}

The proposed algorithm based on the smart integrated adaptive centralized controller is tested on an $11 \mathrm{kV}$ distribution system. Fig. 3 illustrates the topology of distribution network architecture considered as full scale test model where distributed energy resources is conceptualized as a part of the distribution system.

Test system is consisting of a total of 35 buses. The 26 active loads are considered as static impedances in the simulations where: a 2 MVA mini-hydro generation units, a 1 MW PV array panel utilized as main renewable energy resource units, a $500 \mathrm{~kW}$ battery modelled as an energy storage backup/buffer asset and a $500 \mathrm{~kW}$ diesel generator as synchronous energy generation/sustaining asset. Overall, the above distribution enables our test system to constitute a total load of 3.09 MW in peak loading condition (including distribution line losses) where $2.56 \mathrm{MW}$ of RES generations is expected from mini-hydro, $\mathrm{PV}$, battery storage and diesel generator, and $0.53 \mathrm{MW}$ is supplying from main grid, respectively.

Fig. 3 also shows the distribution system is connected to the main grid via a 4 MVA $132 \mathrm{kV} / 11 \mathrm{kV}$ main transformer at the substation number 1530 . The $1 \mathrm{MW}$ PV array as a renewable energy resource and a $500 \mathrm{~kW}$ battery as an energy storage backup are connected via a 2 MVA $0.69 \mathrm{kV} / 11 \mathrm{kV}$ transformer at the substation number 1510 , along with 2 MVA mini-hydro generation and a $500 \mathrm{~kW}$ diesel generator as synchronous energy generation assets connected via 4 MVA $3.3 \mathrm{kV} / 11 \mathrm{kV}$ transformer at the substation number 1531.

\section{A. Test Scenarios}

The proposed algorithm is simulated and tested in the distribution network which is presented in Fig. 3. Sudden disconnection of main grid is simulated to validate the proposed algorithm with different loading conditions such as peak and base loading conditions. The circuit breaker of the main grid is opened at $t=3 \mathrm{~s}$. This scenario is simulated for different loading conditions of the system; during peak and base loading condition main grid is supplied $0.53 \mathrm{MW}$ and $0.31 \mathrm{MW}$ of active power to the distribution network, respectively. For both test scenarios (peak and base loading condition), it has been assumed that PV is generating fixed amount of active power around $1 \mathrm{MW}$ and mini-hydro is generating 1.53 MW into distribution system.

\section{RESULTS AND VALIDATION}

The proposed smart integrated adaptive centralized controller is validated in this section using two test cases of the system which is stated as follow:

A. Microgrid sudden disconnection from the main grid during peak loading condition, and

$B$. Microgrid sudden disconnection from the main grid during base loading condition.

In both cases the islanding operation is simulated by opening the main grid circuit breaker at $t=3 \mathrm{~s}$. These cases were selected to validate the ability of the proposed controller in order to keep system frequency stability, security and power quality of islanded microgrid.

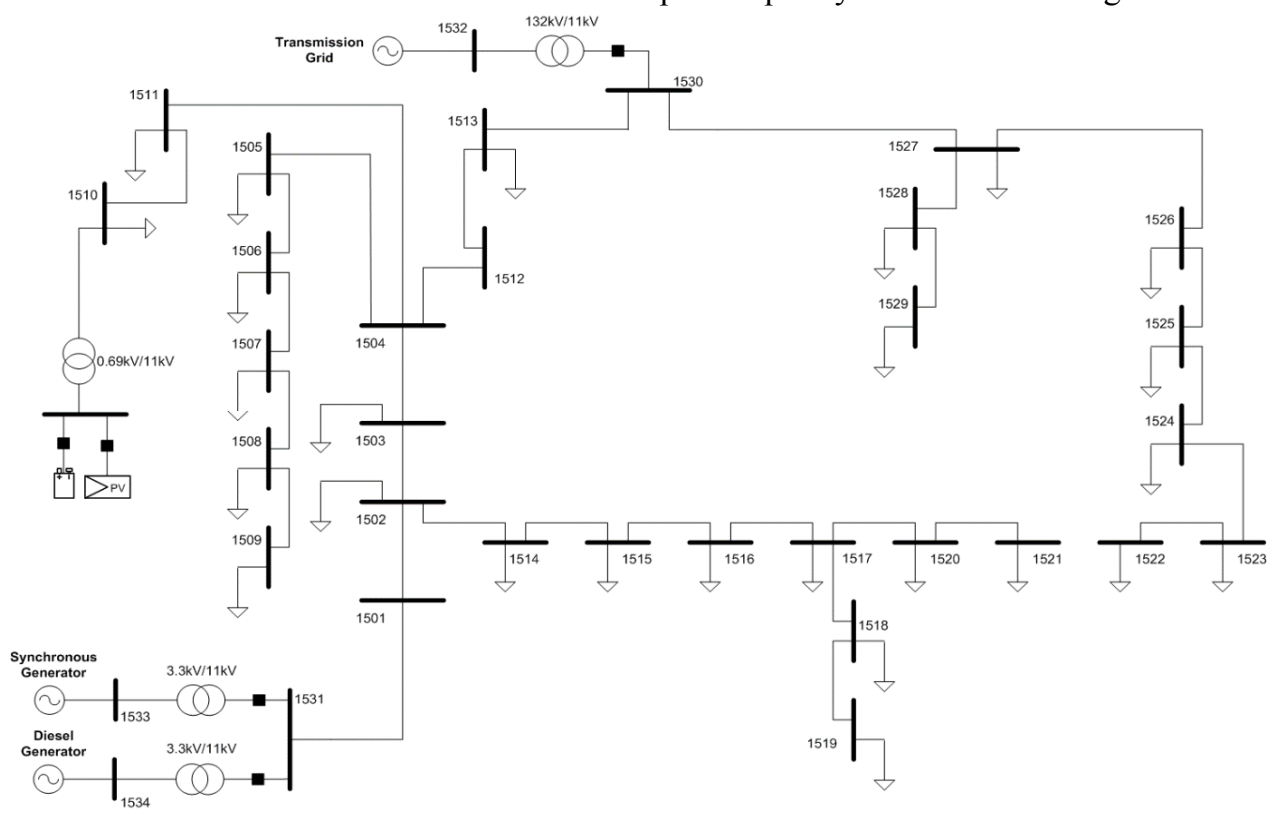

Fig. 3. Distribution network model considered for proposed controller. 
A. Islanding operation in peak loading condition

In this case, the islanding operation of the microgrid is simulated. The total load demand prior to islanding is 3.09 MW (this value is including distribution line losses). The grid and RESs supply the microgrid with $0.53 \mathrm{MW}$ and $2.56 \mathrm{MW}$ of active power, respectively. Subsequent to being islanded, the system frequency begins to decline in response to the excess of load. In this case, the proposed controller detects the frequency decline in islanded microgrid and it will determine the power imbalance amount. Then, the proposed controller will send a required amount of active power to the BESS which is $0.53 \mathrm{MW}$. However, in this study, the maximum amount of active power of BESS that can be injected to microgrid is $0.5 \mathrm{MW}$. Hence, centralized controller will request $0.5 \mathrm{MW}$ active power from BESS and it will start the synchronization process of standby diesel generator. Fig. 4 is presented the frequency response of microgrid. It can be clearly seen that the frequency of microgrid is declined after $t=3$ (s) due to lack of active power. However, it is recovered after injection of active power by BESS. In this case study, system frequency is fallen to $49.35 \mathrm{~Hz}$ and then it is recovered gradually to nominal range.
Active power generation of RESs and main grid prior and after islanding operation is also presented in Fig. 5. This figure is shown that fast response of BESS can be utilized right after islanding detection and it will compensate the lack of active power generation into islanded microgrid. In these figures, synchronization process of diesel generator into islanded microgrid is also shown. The proposed controller will close diesel generator circuit breaker whenever synchronization parameters limit of Table 1 be satisfied.

Fig. 6 is presented system frequency during synchronization process of diesel generator into islanded microgrid. As mentioned, the proposed controller starts the diesel generator right after islanding operation of microgrid at $t=3 \mathrm{~s}$. Then, the diesel generator circuit breaker is commanded by the controller to close at around $t=10 \mathrm{~s}$ when the synchronization parameters limits as specified in Table 1 are satisfied. There is some oscillation in active power and frequency of microgrid after connection of diesel generator and disconnection of BESS. However, this fluctuation is not significant and it is due to changes of power flow in microgrid.

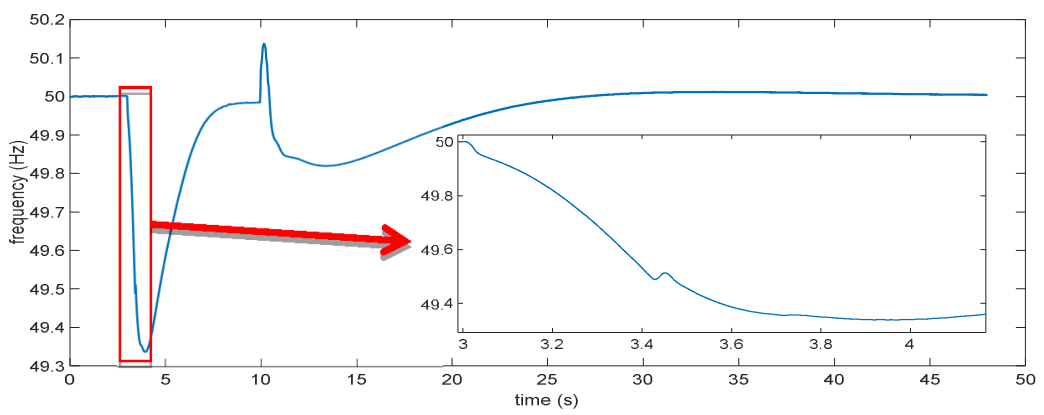

Fig. 4. System frequency response for case $A$



Fig. 5. Active power generation into microgrid for case $A$

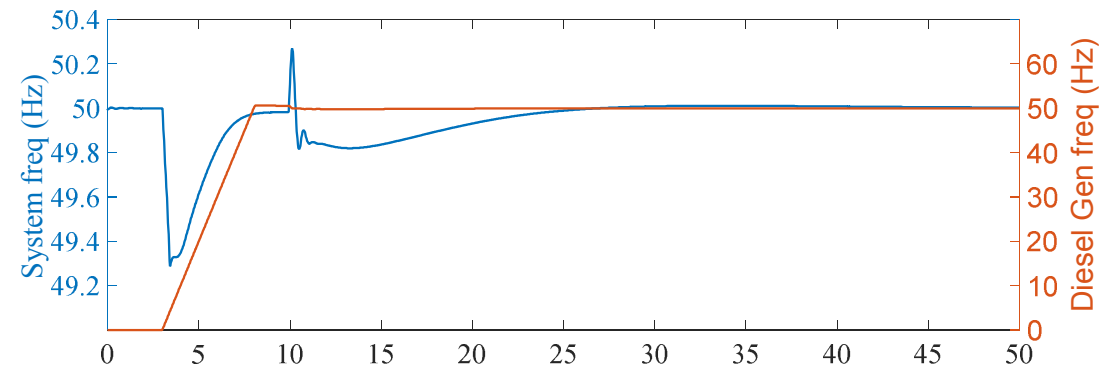

Fig. 6. System frequency during synchronization process 
$B$. Islanding operation in base loading condition

The islanding operation of microgrid for base loading condition is simulated in this case. The total load demand prior to islanding operation is $2.86 \mathrm{MW}$ (this value is including distribution line losses). An active power which is supplied by main grid prior to islanding operation is 0.31 MW in this scenario. Hence, system frequency is declined right after microgrid islanded from main grid at $t=3(\mathrm{~s})$ as illustrated in Fig. 7. Then, the proposed controller will estimate the power imbalance of microgrid and it will send the set point of active power required for the BESS. In this test case, the required power is estimated equal to $0.3 \mathrm{MW}$.

Fig. 8 is shown active power of RESs and grid prior and after islanding operation of microgrid. System frequency during synchronization process of diesel generator into islanded microgrid is also shown in Fig. 9.

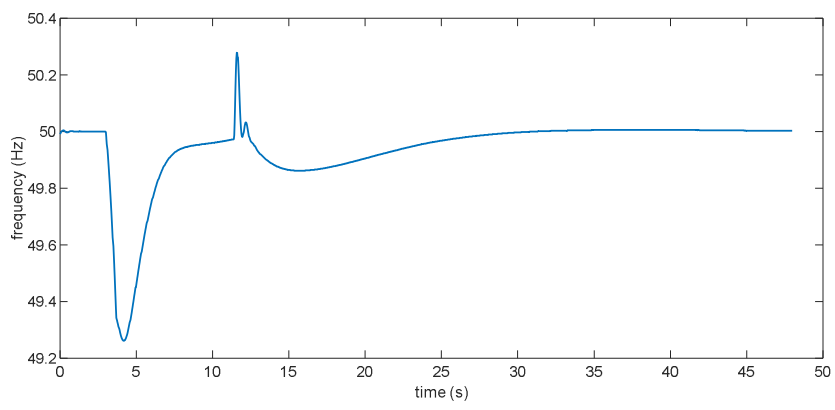

Fig. 7. System frequency response for case $B$

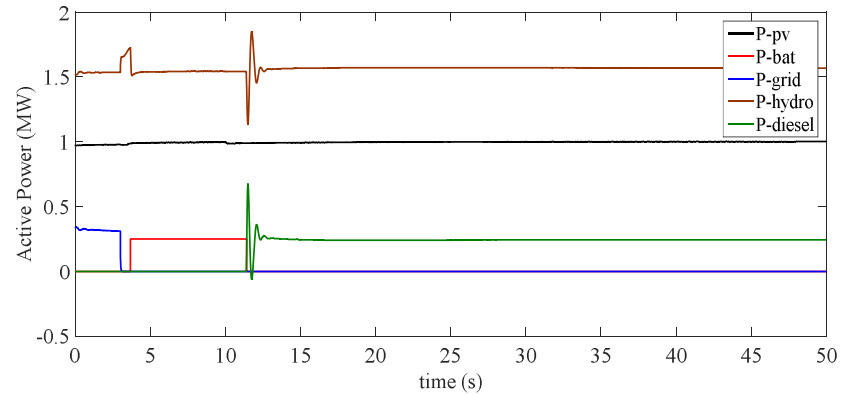

Fig. 8. Active power generation into microgrid for case $B$

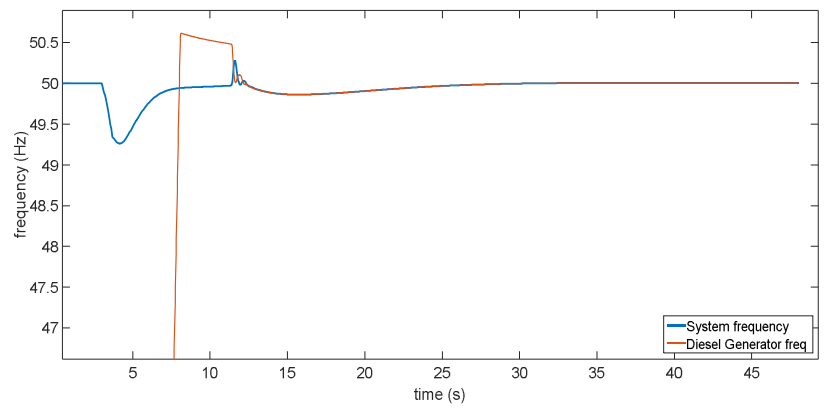

Fig. 9. Synchronization process for case $B$

\section{CONCLUSIONS}

A smart integrated centralized controller is proposed for monitoring and controlling integrated renewable energy sources (RESs), both for intentional or unintentional islanding modes of operation for microgrids. A coordination strategy for RESs is developed for frequency control of microgrid in grid-connected or islanding mode of operations. The performance of proposed centralized controller in terms of a fast frequency response recovery feature is validated in distribution network. The results are demonstrated that the proposed controller is successful in preventing underfrequency load shedding and recovering system frequency in microgrid.

\section{ACKNOWLEDGMENT}

This work was performed as part of the Enhanced Frequency Control Capability (EFCC) Project and was funded though the Network Innovation Competition in GB.

\section{REFERENCES}

[1] D. Wu, F. Tang, T. Dragicevic, J. C. Vasquez, and J. M. Guerrero, "A control architecture to coordinate renewable energy sources and energy storage systems in islanded microgrids," IEEE Transactions on Smart Grid, vol. 6, pp. 1156-1166, 2015.

[2] S. S. Thale and V. Agarwal, "Controller Area Network Assisted Grid Synchronization of a Microgrid With Renewable Energy Sources and Storage," IEEE Transactions on Smart Grid, vol. 7, pp. 1442-1452, 2016.

[3] M. Hossain, H. R. Pota, M. A. Mahmud, and M. Aldeen, "Robust control for power sharing in microgrids with low-inertia wind and PV generators," IEEE Transactions on Sustainable Energy, vol. 6, pp. 1067-1077, 2015.

[4] X. Tang, X. Hu, N. Li, W. Deng, and G. Zhang, "A novel frequency and voltage control method for islanded microgrid based on multienergy storages," IEEE Transactions on Smart Grid, vol. 7, pp. 410-419, 2016.

[5] H. Gao, Y. Chen, Y. Xu, and C.-C. Liu, "Dynamic Load Shedding for an Islanded Microgrid with Limited Generation Resources," IET Generation, Transmission \& Distribution, 2016.

[6] L. Minchala-Avila, L. Garza-Castanon, Y. Zhang, and H. Ferrer, "Optimal Energy Management for Stable Operation of an Islanded Microgrid."

[7] N. L. Diaz, A. C. Luna, J. C. Vasquez, and J. M. Guerrero, "Centralized Control Architecture for Coordination of Distributed Renewable Generation and Energy Storage in Islanded AC Microgrids," IEEE Transactions on Power Electronics.

[8] M. Karimi, P. Wall, H. Mokhlis, and V. Terzija, "A New Centralized Adaptive Under-Frequency Load Shedding Controller for Microgrids based on a Distribution State Estimator."

[9] M. S. Golsorkhi, Q. Shafiee, D. Lu, and J. M. Guerrero, "A Distributed Control Framework for Integrated PhotovoltaicBattery Based Islanded Microgrids," IEEE Transactions on Smart Grid, 2016.

[10] O. Palizban, K. Kauhaniemi, and J. M. Guerrero, "Microgrids in active network management-part II: System operation, power quality and protection," Renewable and Sustainable Energy Reviews, vol. 36, pp. 440-451, 2014.

[11] V. V. Terzija, "Adaptive underfrequency load shedding based on the magnitude of the disturbance estimation," IEEE Transactions on Power Systems, vol. 21, pp. 1260-1266, 2006.

"IEEE Standard for Interconnecting Distributed Resources with Electric Power Systems," IEEE Std 1547-2003, pp. 1-28, 2003. 